\title{
ANÁLISE HISTOLOGICA DE TUMORES MAMARIOS SUBMETIDOS À TERAPIA FOTODINÂMICA
}

\author{
Rainara Moreno Sanches de Almeida ${ }^{1}$ \\ Letícia Corrêa Fontana ${ }^{2}$ \\ André Henrique Correia Pereira ${ }^{3}$ \\ Mirian Aparecida Alves Freitas ${ }^{4}$ \\ Anna Carolina Dentello Rondina Nascimento ${ }^{5}$ \\ Isabelle Ferreira ${ }^{6}$ \\ Juliana Ferreira-Strixino ${ }^{7}$
}

Resumo: A terapia fotodinâmica (TFD) é uma modalidade terapêutica empregada no tratamento de diversas neoplasias malignas. Ela consiste na ação combinada de um composto fotossensível (FS), luz em comprimento de onda adequado e oxigênio molecular, gerando espécies reativas de oxigênio que levam as células tumorais a morte por necrose ou apoptose. O estudo teve como objetivo a análise histológica de tumores mamários, induzidos quimicamente, após a realização TFD. Foram utilizadas 15 ratas da linhagem Sprague-Dawley, divididas em dois grupos, grupo controle (GC - tumor mamário não tratado) e grupo submetido à terapia fotodinâmica (G1 - tumor mamário retirado após $24 \mathrm{~h}$ da realização da TFD). Na análise histológica observaram-se diversas características gerais de malignidade em ambos os grupos. No entanto, o grupo tratado com TFD apresentou uma alta resposta inflamatória e presença de necrose mostrando a eficácia do tratamento no combate ao câncer de mama.

Palavras-chave: Terapia fotodinâmica; Câncer de mama; Histologia.

\footnotetext{
${ }^{1}$ Instituto de Pesquisa e Desenvolvimento (IP\&D) /Universidade do Vale do Paraíba - UNIVAP, Brasil. E-mail: raimsanches@hotmail.com.

2 Instituto de Pesquisa e Desenvolvimento (IP\&D) /Universidade do Vale do Paraíba - UNIVAP, Brasil. E-mail: leticia.fontana@yahoo.com.

3 Instituto de Pesquisa e Desenvolvimento (IP\&D) /Universidade do Vale do Paraíba - UNIVAP, Brasil. E-mail: andre_gcp@hotmail.com.

4 Instituto de Pesquisa e Desenvolvimento (IP\&D) /Universidade do Vale do Paraíba - UNIVAP, Brasil. E-mail: mirianafreitas@hotmail.com.

5 Instituto de Pesquisa e Desenvolvimento (IP\&D) /Universidade do Vale do Paraíba - UNIVAP, Brasil. E-mail: carol_dentello@hotmail.com.

${ }^{6}$ Universidade Paulista - UNIP, Brasil. E-mail: iferreira_16@hotmail.com.

7 Instituto de Pesquisa e Desenvolvimento (IP\&D) /Universidade do Vale do Paraíba - UNIVAP, Brasil. E-mail: juferreira@univap.br.
} 